KYUNGPOOK Math. J. 55(2015), 169-180

http://dx.doi.org/10.5666/KMJ.2015.55.1.169

pISSN 1225-6951 eISSN 0454-8124

(c) Kyungpook Mathematical Journal

\title{
On the Braid Index of Kanenobu Knots
}

\author{
HiDEO TAKIOKA \\ Osaka City University Advanced Mathematical Institute, Sugimoto, Sumiyoshi-ku, \\ Osaka 558-8585, Japan \\ e-mail : takioka@sci.osaka-cu.ac.jp
}

Abstract. We study the braid indices of the Kanenobu knots. It is known that the Kanenobu knots have the same HOMFLYPT polynomial and the same Khovanov-Rozansky homology. The MFW inequality is known for giving a lower bound of the braid index of a link by applying the HOMFLYPT polynomial. Therefore, it is not easy to determine the braid indices of the Kanenobu knots. In our previous paper, we gave upper bounds and sharper lower bounds of the braid indices of the Kanenobu knots by applying the 2-cable version of the zeroth coefficient HOMFLYPT polynomial. In this paper, we give sharper upper bounds of the braid indices of the Kanenobu knots.

\section{Introduction}

Every oriented link in the 3 -sphere is presented as a closed braid [1]. The braid index, denoted by braid $(L)$, of an oriented link $L$ is the minimum number of strings needed for $L$ to be presented as a closed braid, which is an invariant of the isotopy type of $L$. The MFW inequality is known for giving a lower bound of $\operatorname{braid}(L)$ by applying the HOMFLYPT polynomial $P(L)=P(L ; v, z)$ in $\mathbb{Z}\left[v^{ \pm 1}, z^{ \pm 1}\right]([2],[9])$ :

$$
\frac{1}{2} v \text {-span } P(L)+1 \leq \operatorname{braid}(L)
$$

where $v$-span $P(L)$ is the difference between the maximum and minimum degrees of $P(L)$ on the variable $v$, denoted by $v$-maxdeg $P(L)$ and $v$-mindeg $P(L)$, respectively. The HOMFLYPT polynomial $P(L)$ is an invariant of the isotopy type of $L$, which is computed by the following recursive formula ([3], [7], [10]):

$$
P(U)=1 \text { for the unknot } U \text {; }
$$

Received November 8, 2013; revised March 28, 2014; accepted July 14, 2014.

2010 Mathematics Subject Classification: 57M25, 57M27.

Key words and phrases: braid index, HOMFLYPT polynomial, Kanenobu knot, 2-cable knot. 


$$
v^{-1} P\left(L_{+}\right)-v P\left(L_{-}\right)=z P\left(L_{0}\right),
$$

where $L_{+}, L_{-}$, and $L_{0}$ are three oriented links which are identical except near one point as shown in Fig. 1. In this paper, we study the braid index of the Kanenobu
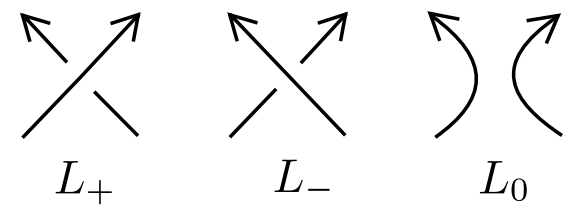

Figure 1: Skein triple.

knot $k(n)$ for $n \geq 0$ as shown in Fig. 2. It is known that the Kanenobu knots have the same HOMFLYPT polynomial and the same Khovanov-Rozansky homology $([4],[8]):$

$$
P(k(n))=\left(v^{-2}-1+v^{2}-z^{2}\right)^{2} \text { for any } n .
$$

By (1.1), we have

$$
\operatorname{braid}(k(n)) \geq 5 \text { for any } n \text {. }
$$

Therefore, it is not easy to determine $\operatorname{braid}(k(n))$. In the previous paper [11], we gave an upper bound and a sharper lower bound of $\operatorname{braid}(k(n))$ by applying the 2-cable version of the zeroth coefficient HOMFLYPT polynomial as follows:

$$
\begin{cases}\operatorname{braid}(k(n))=5 & \text { if } n=0,1 \\ n+3 \leq \operatorname{braid}(k(n)) \leq 2 n+3 & \text { if } n \geq 2 .\end{cases}
$$

In this paper, we give a sharper upper bound of braid $(k(n))$ for $n \geq 2$ and determine braid $(k(2))$ as follows:

Theorem 1.1. Let $k(n)$ be the Kanenobu knot for $n \geq 0$. Then we have

$$
\begin{cases}\operatorname{braid}(k(n))=5 & \text { if } n=0,1,2, \\ n+3 \leq \operatorname{braid}(k(n)) \leq 2 n+1 & \text { if } n \geq 3\end{cases}
$$

\section{Proof of Theorem 1.1}

In this section, we give an upper bound of $\operatorname{braid}(k(n))$ for $n \geq 2$.

Proof of Theorem 1.1.

First, we transform the Kanenobu knot $k(n)$ as shown in Fig. 3 I-VII and set the axis perpendicular to this paper through the point $\mathbf{x}$ as shown in Fig. 3 VII. Next, 


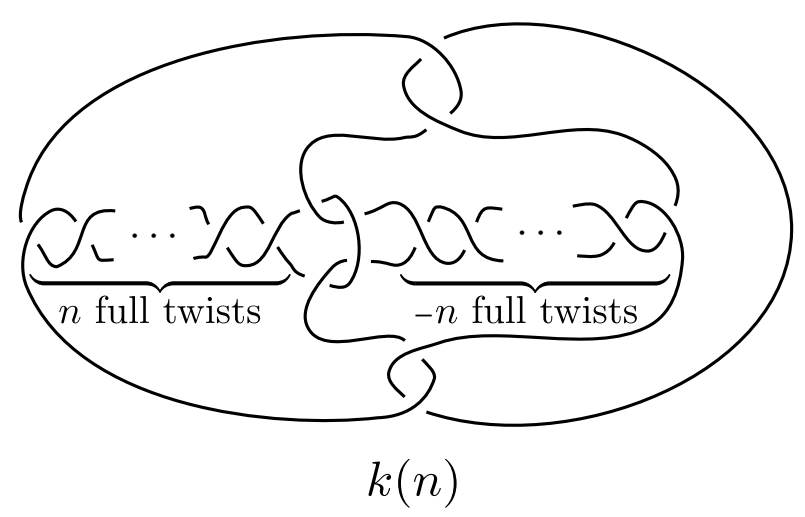

Figure 2: Kanenobu knot $k(n)$.

we turn over strands with counterclockwise orientation with the axis as shown in Fig. 4 VIII and transform $k(n)$ as shown in Fig. 4 VIII-XIII. Finally, we see that an upper bound of $\operatorname{braid}(k(n))$ is $2 n+1$.

Remark 2.1. In Theorem 1.1, we give a lower bound of braid $(k(3))$ by applying the 2-cable version of the zeroth coefficient HOMFLYPT polynomial. However, we cannot give a sharper lower bound of braid $(k(3))$ from the entire HOMFLYPT polynomial of the $(2, q)$-cable link, denoted by $k(3)^{(2, q)}$, of $k(3)$ for any $q \in \mathbb{Z}$. In fact, we have $P\left(k(3)^{(2,0)}\right)$ and $P\left(k(3)^{(2,1)}\right)$ as shown in Tables 1 and 2 . Here the HOMFLYPT polynomial $P(L ; v, z)$ is given as a matrix of coefficients $\left(p_{i j}\right)$, where $P(L ; v, z)=\sum p_{i j} v^{i} z^{j}$, with the range of $i$ and $j$ indicated at the side.

\begin{tabular}{|c|c|c|c|c|c|c|c|c|c|c|c|c|}
\hline-1 & 1 & 3 & 5 & 7 & 9 & 11 & 13 & 15 & 17 & 19 & 21 & \\
\hline 0 & -17 & -236 & -1058 & -2214 & -2507 & -1652 & -651 & -151 & -19 & -1 & 0 & -11 \\
\hline 1 & 59 & 700 & 3251 & 7434 & 9534 & 7387 & 3591 & 1103 & 208 & 22 & 1 & -9 \\
\hline-5 & -84 & -668 & -2874 & -6674 & -8823 & -7023 & -3486 & -1087 & -207 & -22 & -1 & -7 \\
\hline 14 & 95 & 245 & 500 & 1031 & 1442 & 1147 & 519 & 133 & 18 & 1 & 0 & -5 \\
\hline-26 & -126 & -168 & 102 & 426 & 378 & 150 & 28 & 2 & 0 & 0 & 0 & -3 \\
\hline 35 & 143 & 237 & 171 & 30 & -20 & -9 & -1 & 0 & 0 & 0 & 0 & -1 \\
\hline-35 & -143 & -237 & -171 & -30 & 20 & 9 & 1 & 0 & 0 & 0 & 0 & 1 \\
\hline 26 & 126 & 168 & -102 & -426 & -378 & -150 & -28 & -2 & 0 & 0 & 0 & 3 \\
\hline-14 & -95 & -245 & -500 & -1031 & -1442 & -1147 & -519 & -133 & -18 & -1 & 0 & 5 \\
\hline 5 & 84 & 668 & 2874 & 6674 & 8823 & 7023 & 3486 & 1087 & 207 & 22 & 1 & 7 \\
\hline-1 & -59 & -700 & -3251 & -7434 & -9534 & -7387 & -3591 & -1103 & -208 & -22 & -1 & 9 \\
\hline 0 & 17 & 236 & 1058 & 2214 & 2507 & 1652 & 651 & 151 & 19 & 1 & 0 & 11 \\
\hline
\end{tabular}

Table 1: $P\left(k(3)^{(2,0)}\right)$. 


\begin{tabular}{|c|c|c|c|c|c|c|c|c|c|c|c|c|}
\hline 0 & 2 & 4 & 6 & 8 & 10 & 12 & 14 & 16 & 18 & 20 & 22 & \\
\hline-2 & -85 & -685 & -2262 & -3840 & -3729 & -2185 & -785 & -169 & -20 & -1 & 0 & -10 \\
\hline 11 & 264 & 2046 & 7151 & 13368 & 14762 & 10195 & 4525 & 1291 & 229 & 23 & 1 & -8 \\
\hline-26 & -300 & -1865 & -6340 & -12117 & -13765 & -9740 & -4405 & -1274 & -228 & -23 & -1 & -6 \\
\hline 48 & 216 & 490 & 1026 & 1909 & 2260 & 1564 & 636 & 150 & 19 & 1 & 0 & -4 \\
\hline-66 & -236 & -172 & 339 & 700 & 504 & 176 & 30 & 2 & 0 & 0 & 0 & -2 \\
\hline 45 & 172 & 248 & 136 & -3 & -30 & -10 & -1 & 0 & 0 & 0 & 0 & 0 \\
\hline 10 & 29 & 11 & -35 & -33 & -10 & -1 & 0 & 0 & 0 & 0 & 0 & 2 \\
\hline-40 & -110 & -4 & 237 & 274 & 126 & 26 & 2 & 0 & 0 & 0 & 0 & 4 \\
\hline 34 & 121 & 245 & 526 & 878 & 818 & 417 & 117 & 17 & 1 & 0 & 0 & 6 \\
\hline-21 & -216 & -1197 & -3466 & -5443 & -4942 & -2717 & -919 & -187 & -21 & -1 & 0 & 8 \\
\hline 10 & 205 & 1346 & 3900 & 5934 & 5228 & 2808 & 934 & 188 & 21 & 1 & 0 & 10 \\
\hline-2 & -68 & -449 & -1204 & -1626 & -1222 & -533 & -134 & -18 & -1 & 0 & 0 & 12 \\
\hline
\end{tabular}

Table 2: $P\left(k(3)^{(2,1)}\right)$

We see that

$$
\begin{aligned}
& v \text {-maxdeg } P\left(k(3)^{(2,0)}\right)=11, v \text {-mindeg } P\left(k(3)^{(2,0)}\right)=-11, \\
& v \text {-maxdeg } P\left(k(3)^{(2,1)}\right)=12, v \text {-mindeg } P\left(k(3)^{(2,1)}\right)=-10 .
\end{aligned}
$$

By (1.2), we have

$$
P\left(k(3)^{(2, q)}\right)= \begin{cases}v^{2} P\left(k(3)^{(2, q-2)}\right)+v z P\left(k(3)^{(2, q-1)}\right) & \text { if } q \geq 2, \\ v^{-2} P\left(k(3)^{(2, q+2)}\right)-v^{-1} z P\left(k(3)^{(2, q+1)}\right) & \text { if } q \leq-1 .\end{cases}
$$

We see inductively that

$$
v \text {-span } P\left(k(3)^{(2, q)}\right) \leq 22 \text { for any } q \in \mathbb{Z} .
$$

By (1.1), we have

$$
\frac{1}{2} v \text {-span } P\left(k(3)^{(2, q)}\right)+1 \leq \operatorname{braid}\left(k(3)^{(2, q)}\right) .
$$

By Theorem 1 in [12], we have

$$
\operatorname{braid}\left(k(3)^{(2, q)}\right)=2 \operatorname{braid}(k(3)) .
$$

Therefore, we have

$$
6 \leq \operatorname{braid}(k(3)) .
$$

Here we discuss how to compute $P\left(k(3)^{(2,0)}\right)$ and $P\left(k(3)^{(2,1)}\right)$ by Kodama's KNOT program [6]. Since $k(3)^{(2,0)}$ and $k(3)^{(2,1)}$ have large crossing numbers, we cannot apply Kodama's KNOT program to these links directly. We use a skein relation for the HOMFLYPT polynomial of 2-cable links given in [5]. Let $L\left(t_{+}\right), L\left(t_{-}\right), L\left(e_{+}\right)$, $L\left(e_{-}\right), L\left(f_{+}\right), L\left(f_{0}\right)$, and $L\left(f_{-}\right)$be oriented links identical outside a ball and inside 
are 8-end tangles $t_{+}, t_{-}, e_{+}, e_{-}, f_{+}, f_{0}$, and $f_{-}$as shown in Fig. 5, respectively. We call the ordered set of links $\left(L\left(t_{+}\right), L\left(t_{-}\right), L\left(e_{+}\right), L\left(e_{-}\right), L\left(f_{+}\right), L\left(f_{0}\right), L\left(f_{-}\right)\right)$a double skein 7-tuple. We denote the HOMFLYPT polynomial of the link $L(s)$ by $P(s)$, where $s$ is one of these tangles. Then we have

$$
\begin{aligned}
& v^{-5} P\left(t_{+}\right)+v^{5} P\left(t_{-}\right) \\
= & v^{-3} P\left(e_{+}\right)+v^{3} P\left(e_{-}\right)+\left(v^{-3} P\left(f_{+}\right)+\left(v^{-1}+v\right) P\left(f_{0}\right)+v^{3} P\left(f_{-}\right)\right) z^{2} .
\end{aligned}
$$

Let $k(a,-b ; c)$ and $T(d)$ be two oriented links as shown in Fig. 6, where 8-end tangles labelled $a$ and $-b$ are $a$ double full twists and $-b$ double full twists for $a, b \geq$ 0 , respectively and 4-end tangles labelled $c$ and $d$ are $c$ half twists and $d$ half twists for $c, d \in \mathbb{Z}$, respectively. Since we can compute $P\left(k(3)^{(2,1)}\right)$ in the same way as $P\left(k(3)^{(2,0)}\right)$, we only compute $P\left(k(3)^{(2,0)}\right)$. We apply $(2.1)$ to $k(3)^{(2,0)}=k(3,-3 ; 0)$ as shown in Figs. 7 and 8 . Then we obtain the following double skein 7 -tuple:

$$
\begin{aligned}
& (k(3,-1 ;-4), k(3,-3 ; 0), k(3,-2 ; 0), k(3,-2 ;-4), T(5) \sqcup T(-1), \\
& T(4) \sqcup T(-2), T(3) \sqcup T(-3)) .
\end{aligned}
$$

We can compute $P(T(5) \sqcup T(-1)), P(T(4) \sqcup T(-2))$, and $P(T(3) \sqcup T(-3))$ by Kodama's KNOT program. However, since $k(3,-1 ;-4), k(3,-2 ; 0)$, and $k(3,-2 ;-4)$ have still large crossing numbers, we cannot apply Kodama's KNOT program to these links directly. In the case of $k(a,-b ; c)$, we obtain the following double skein 7-tuples:

$$
\begin{aligned}
& (k(a,-b ; c), k(a-2,-b ; c+4), k(a-1,-b ; c+4), k(a-1,-b ; c), \\
& T(x+1) \sqcup T(y+1), T(x) \sqcup T(y), T(x-1) \sqcup T(y-1)),
\end{aligned}
$$

where $a, b, c, x$, and $y$ are integers satisfying $a \geq 2, b \geq 0$, and $x+y=4 a-4 b+c-2$,

$$
\begin{aligned}
& (k(a,-b+2 ; c-4), k(a,-b ; c), k(a,-b+1 ; c), k(a,-b+1 ; c-4), \\
& T(x+1) \sqcup T(y+1), T(x) \sqcup T(y), T(x-1) \sqcup T(y-1)),
\end{aligned}
$$

where $a, b, c, x$, and $y$ are integers satisfying $a \geq 0, b \geq 2$, and $x+y=4 a-4 b+c+2$. In order to compute $P(k(3,-1 ;-4))$, we apply $(2.2)$ as follows:

$$
\begin{aligned}
& (k(3,-1 ;-4), k(1,-1 ; 0), k(2,-1 ; 0), k(2,-1 ;-4), T(3) \sqcup T(1), \\
& T(2) \sqcup T(0), T(1) \sqcup T(-1)) .
\end{aligned}
$$

We can compute $P(k(1,-1 ; 0)), P(T(3) \sqcup T(1)), P(T(2) \sqcup T(0))$, and $P(T(1) \sqcup$ $T(-1))$ by Kodama's KNOT program. Since $k(2,-1 ; 0)$ and $k(2,-1 ;-4)$ have still large crossing numbers, we apply (2.2) as follows:

$$
(k(2,-1 ; 0), k(0,-1 ; 4), k(1,-1 ; 4), k(1,-1 ; 0), T(3) \sqcup T(1),
$$




$$
\begin{aligned}
& T(2) \sqcup T(0), T(1) \sqcup T(-1)) ; \\
& (k(2,-1 ;-4), k(0,-1 ; 0), k(1,-1 ; 0), k(1,-1 ;-4), T(-1) \sqcup T(1), \\
& T(-2) \sqcup T(0), T(-3) \sqcup T(-1)) .
\end{aligned}
$$

Since we can compute $P(k(1,-1 ; 0))$ and $P(k(1,-1 ; 1))$ by Kodama's KNOT program, we obtain $P(k(1,-1 ; q))$ by applying (1.2) inductively as follows:

$$
P(k(1,-1 ; q))= \begin{cases}v^{2} P(k(1,-1 ; q-2))+v z P(k(1,-1 ; q-1)) & \text { if } q \geq 2, \\ v^{-2} P(k(1,-1 ; q+2))-v^{-1} z P(k(1,-1 ; q+1)) & \text { if } q \leq-1 .\end{cases}
$$

We can compute the HOMFLYPT polynomials of the remaining links with small crossing numbers by Kodama's KNOT program. Thus, we can compute $P(k(3,-1 ;-4))$. Since we can compute $P(k(3,-2 ; 0))$ and $P(k(3,-2 ;-4))$ in the same way as $P(k(3,-1 ;-4))$, we can finally compute $P\left(k(3)^{(2,0)}\right)$.

Question 2.2. $\operatorname{braid}(k(3))=6,7$ ?

Acknowledgments. The author would like to thank Professor Taizo Kanenobu and Professor Akio Kawauchi for many helpful advices and comments. He also would like to thank the members of Friday Seminar on Knot Theory in Osaka City University.

\section{References}

[1] J. W. Alexander, A lemma on systems of knotted curves, Proc. Nat. Acad. Sci. U. S. A., 9(1923), 93-95.

[2] J. Franks and R. F. Williams, Braids and the Jones Polynomial, Trans. Amer. Math. Soc., 303(1987), 97-108.

[3] P. Freyd, D. Yetter, J. Hoste, W. B. R. Lickorish, K. Millett, and A. Ocneanu, A new polynomial invariant of knots and links, Bull. Amer. Math. Soc., 12(1985), 239-246.

[4] T. Kanenobu, Infinitely many knots with the same polynomial invariant, Proc. Amer. Math. Soc., 97(1986), 158-162.

[5] T. Kanenobu, A skein relation for the HOMFLYPT polynomials of two-cable links, Algebr. Geom. Topol., 7(2007), 1211-1232.

[6] K. Kodama, http://www.math.kobe-u.ac.jp/HOME/kodama/knot.html

[7] W. B. R. Lickorish and K. Millett, A polynomial invariant of oriented links, Topology, 26(1987), 107-141.

[8] A. Lobb, The Kanenobu knots and Khovanov-Rozansky homology, Proc. Amer. Math. Soc., 142(2014), 1447-1455.

[9] H. R. Morton, Seifert circles and knot polynomials, Math. Proc. Cambridge Philos. Soc., 99(1986), 107-109. 
[10] J. H. Przytycki and P. Traczyk, Invariants of links of Conway type, Kobe J. Math., 4(1987), 115-139.

[11] H. Takioka, The zeroth coefficient HOMFLYPT polynomial of a 2-cable knot, J. Knot Theory Ramifications, 22(2)(2013), 1350001.

[12] R. F. Williams, The braid index of generalized cables, Pacific J. Math., 155(1992), 369-375. 

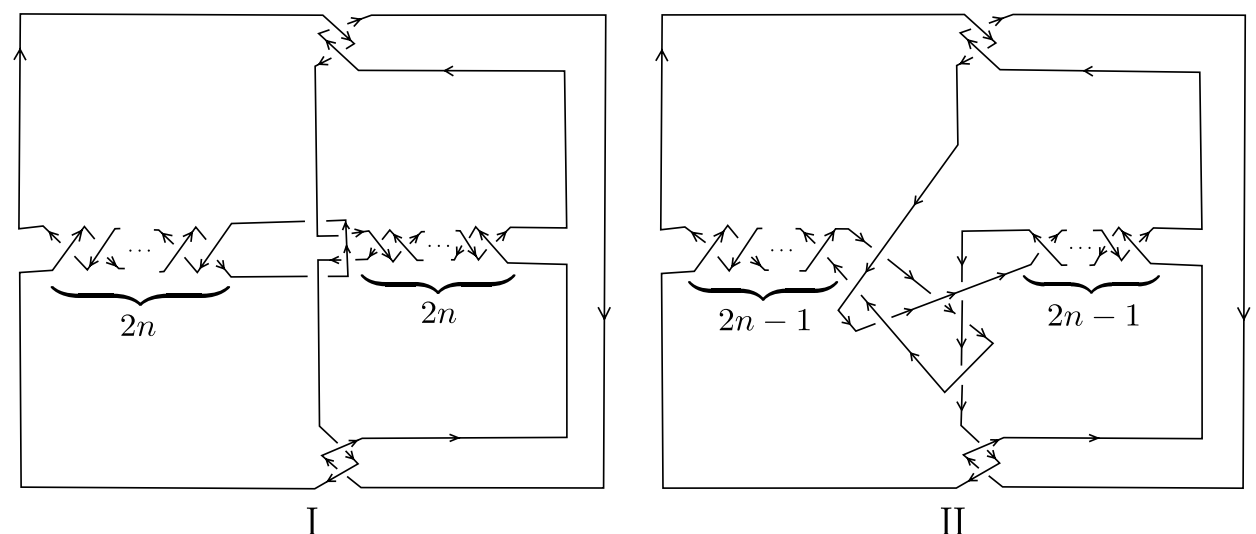

II
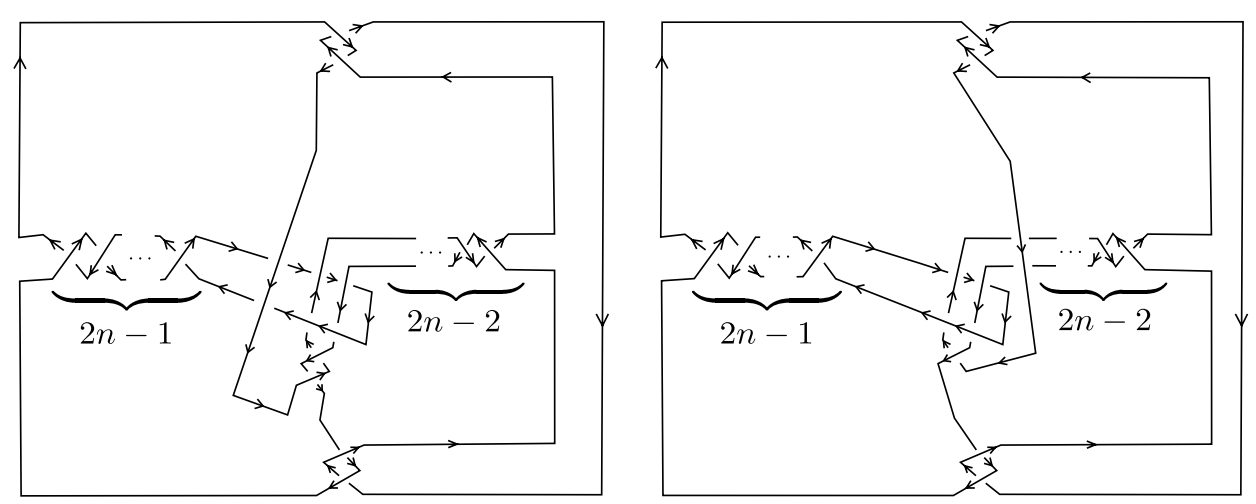

IV
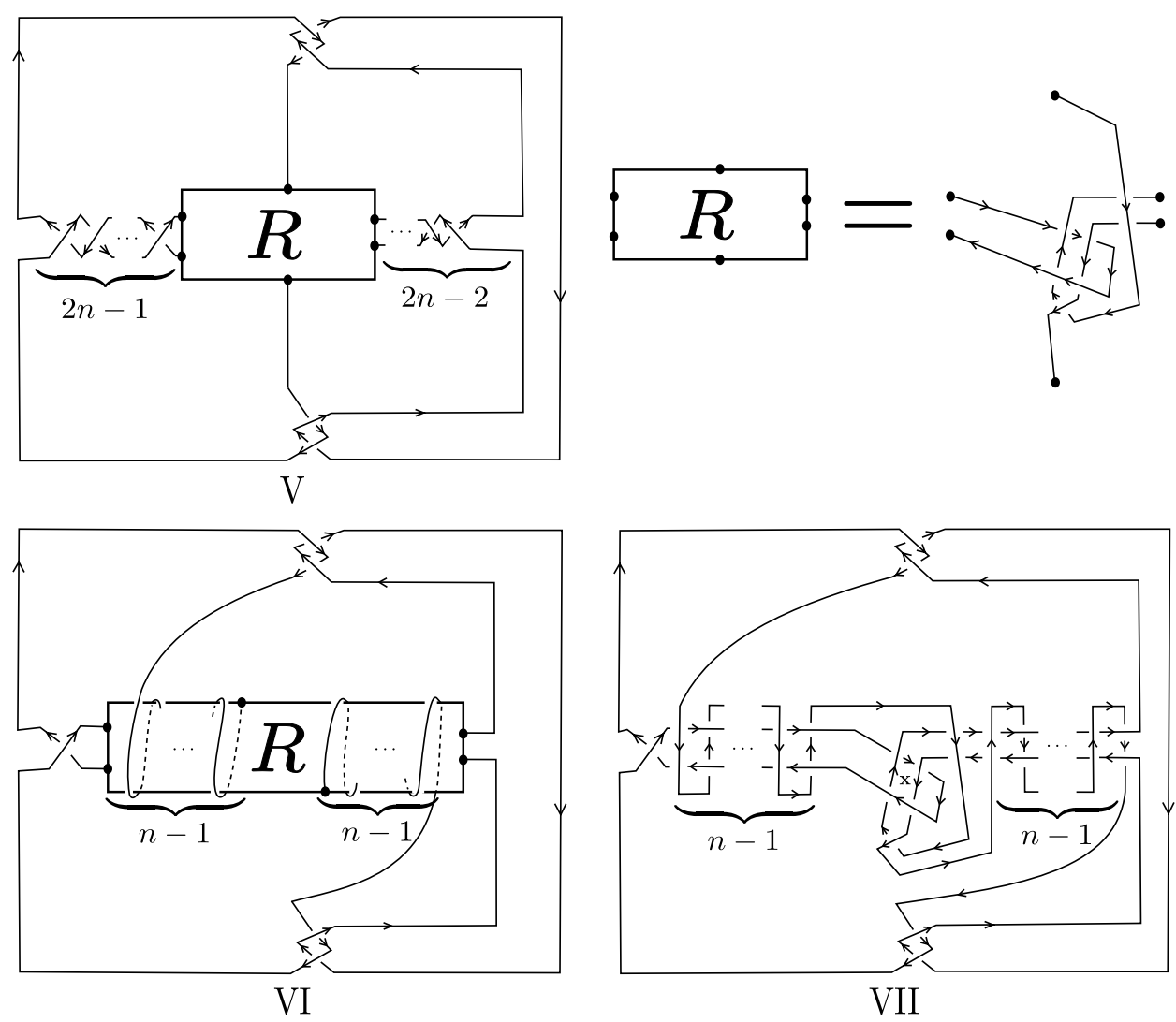

Figure 3: Transformations I-VII. 

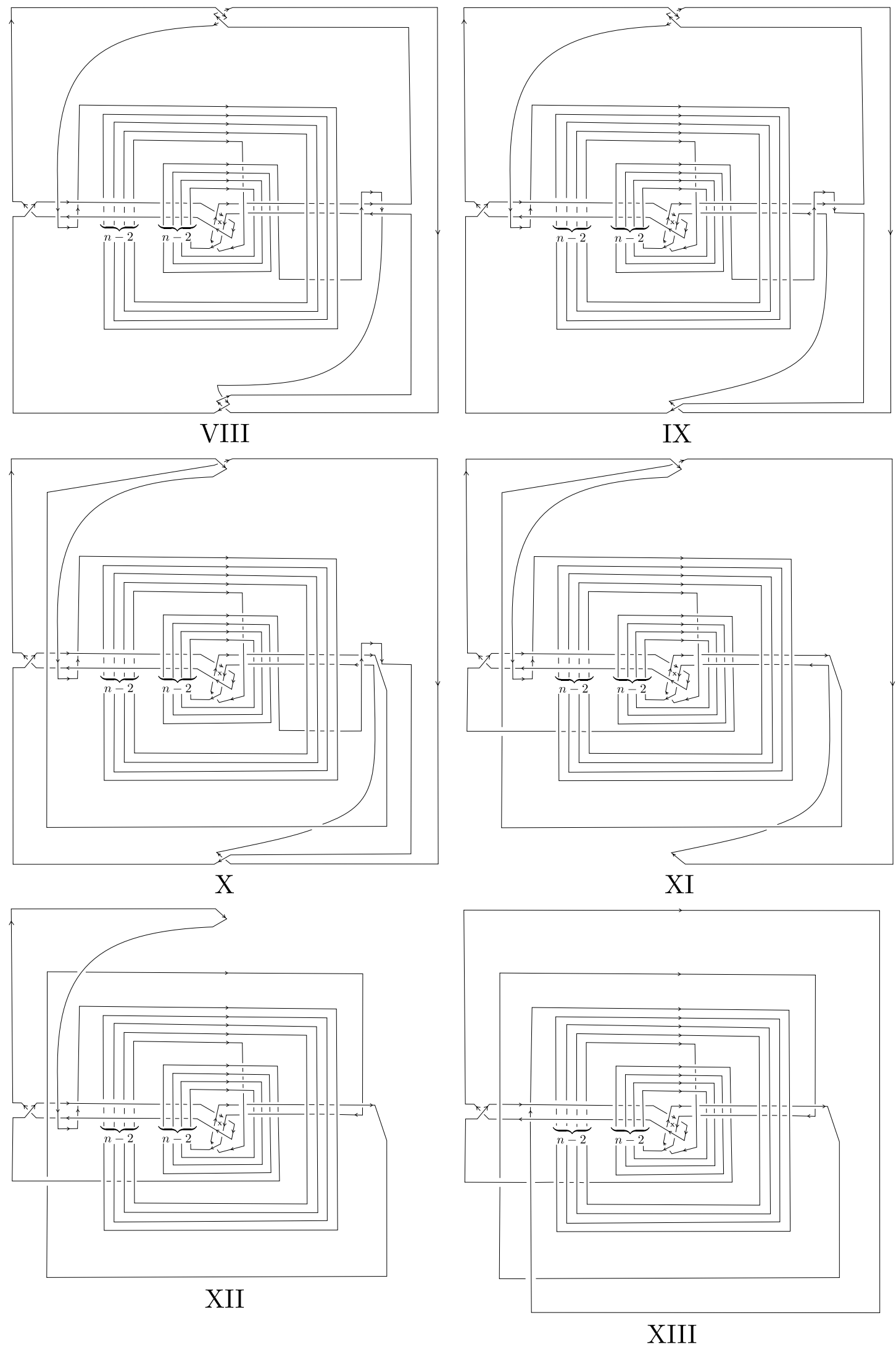

Figure 4: Transformations VIII-XIII. 

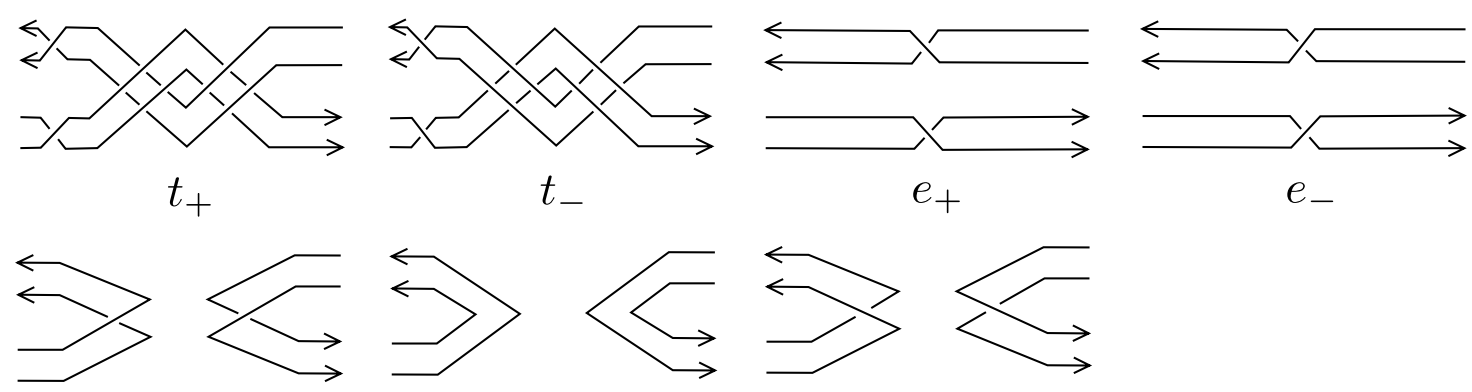

$f_{+}$

$f_{0}$

$f_{-}$

Figure 5: 8-end tangles $t_{+}, t_{-}, e_{+}, e_{-}, f_{+}, f_{0}$, and $f_{-}$.
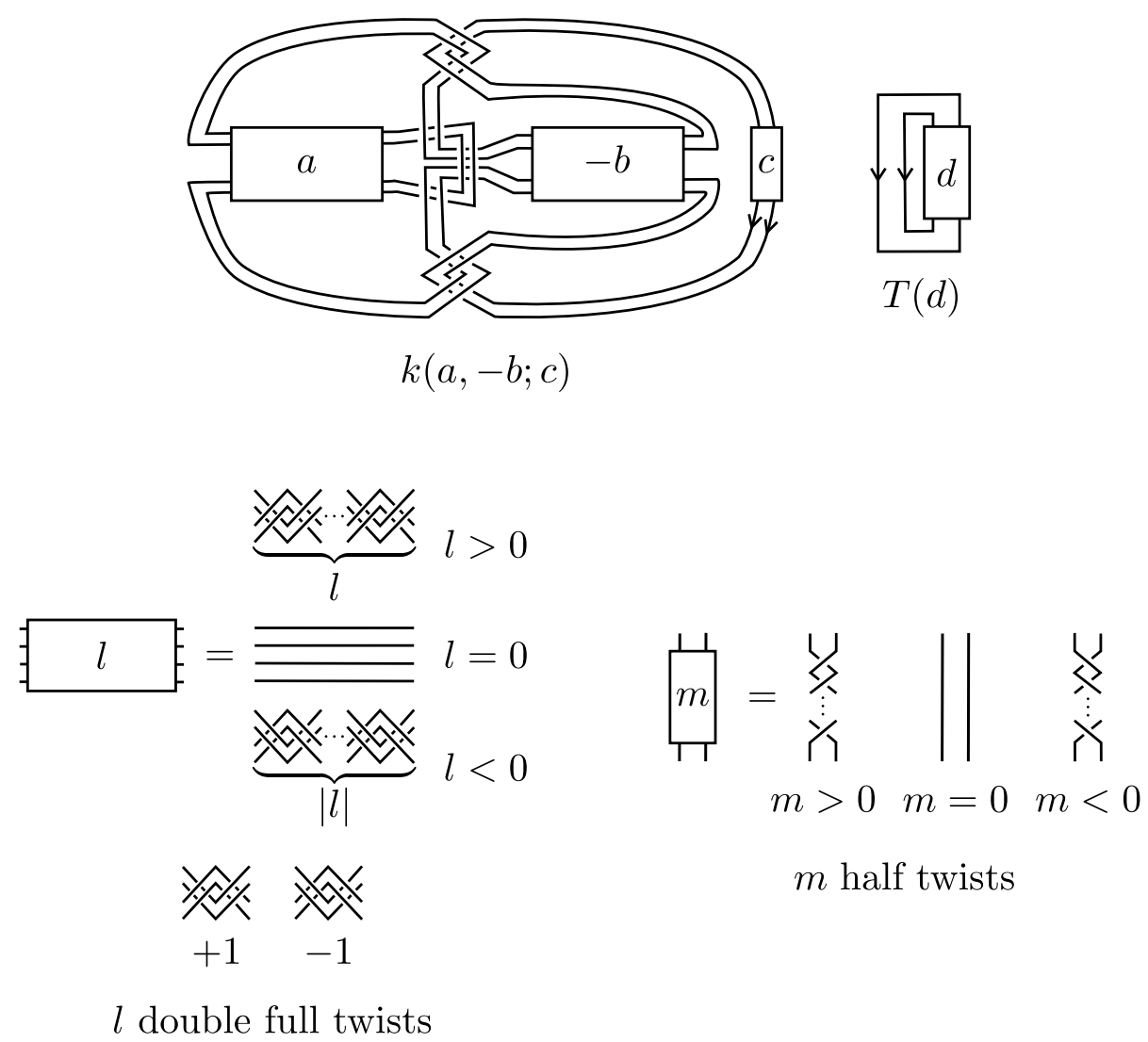

Figure 6: $k(a,-b ; c)$ and $T(d)$. 

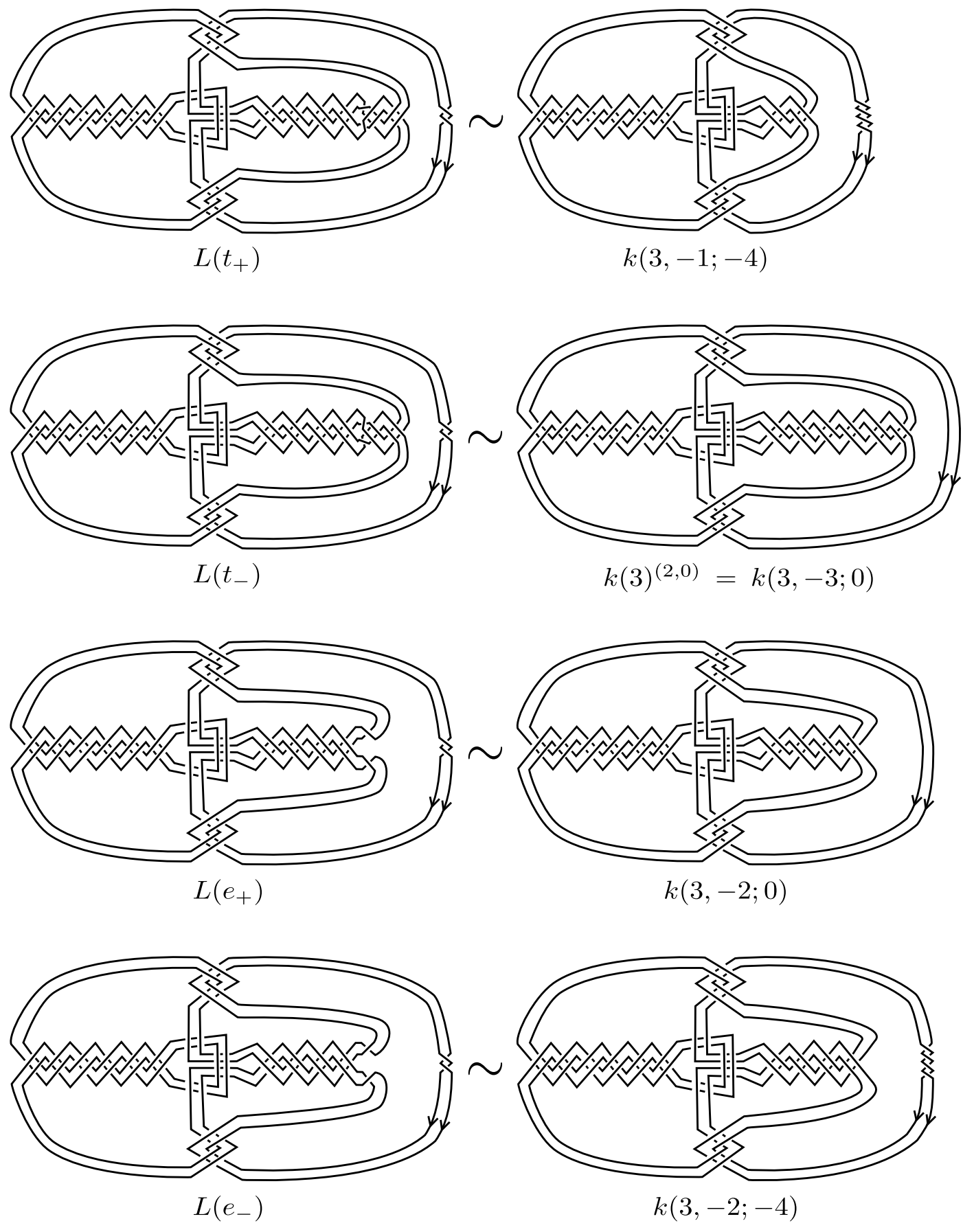

Figure 7: $L\left(t_{+}\right), L\left(t_{-}\right), L\left(e_{+}\right)$, and $L\left(e_{-}\right)$. 

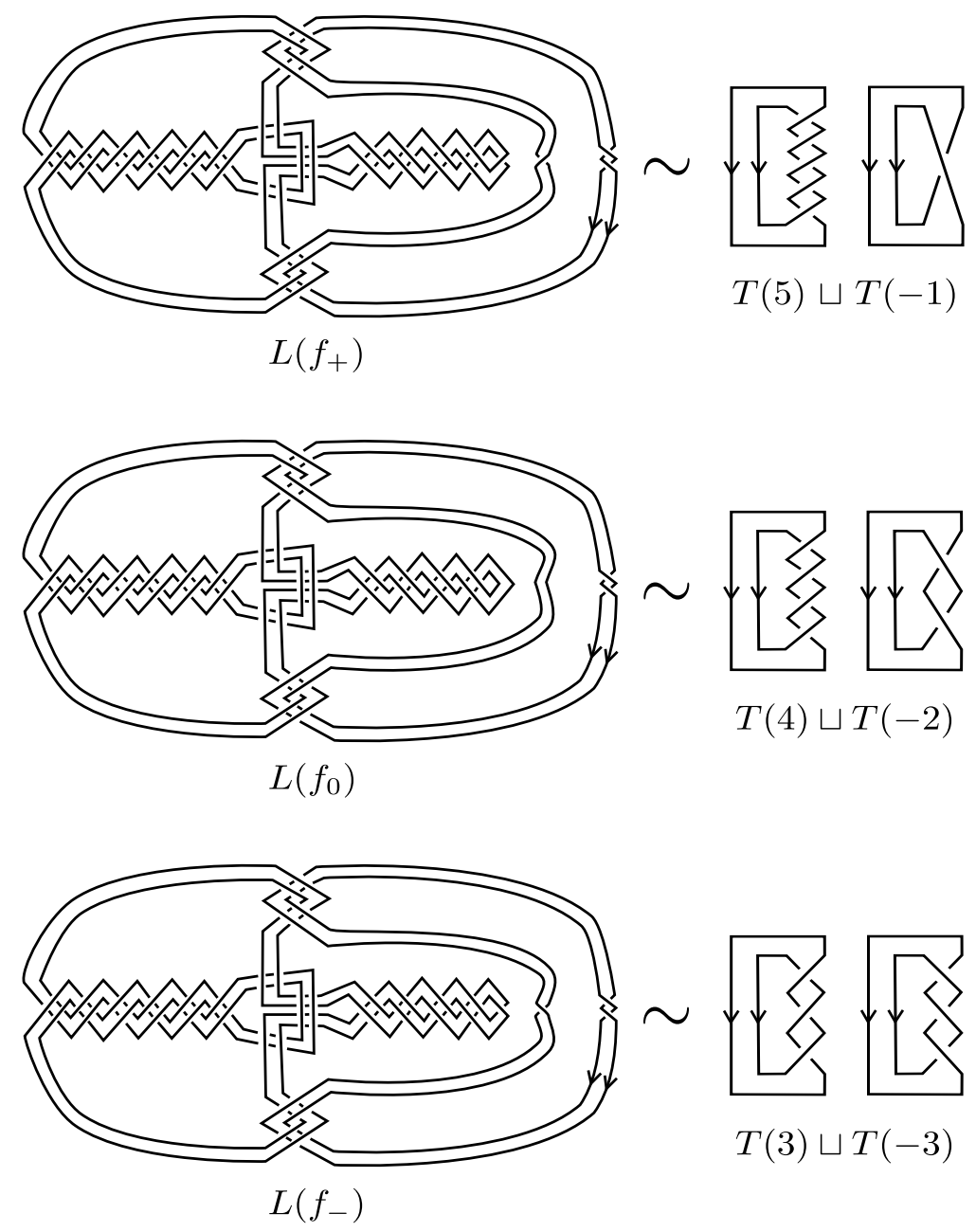

Figure 8: $L\left(f_{+}\right), L\left(f_{0}\right)$, and $L\left(f_{-}\right)$. 